\title{
Moving from Research to Program- The Egyptian Postabortion Care Initiative
}

In settings where abortion is legally restricted and socially sanctioned, the medical treatment of women who have had unsafe or incomplete abortions is often a willfully neglected service. Research conducted in the 1990s brought attention to the low quality of care and inhumane treatment that many patients receive as a result of this neglect. ${ }^{1}$ The concept of postabortion care was central to this research, which highlighted three essential services for providers to offer women who seek care for an incomplete abortion: emergency medical treatment of complications, family planning counseling and services, and referral for other reproductive health care needs. By clearly positioning the problem within the health care domain-as opposed to religious, legal or social arenas-advocates of postabortion care have created a less-volatile atmosphere for research and program development.

Yet, tension continues to surround activities concerned with abortion. As postabortion care programs expand out of the pilot phase and scale up their activities as elements of larger, ongoing health care programs, they test the limits of public officials' support. The literature offers little evidence of how successfully these programs have managed resistance associated with the politics of abortion and grown from pilot studies to national programs.

An additional difficulty confronting postabortion programs concerns the supply of manual vacuum aspiration instruments. The overwhelming body of evidence favors the use of manual vacuum aspiration for the treatment of incomplete abortion at an early gestational age, ${ }^{2}$ as a result, it has become the standard for postabortion care. ${ }^{3}$ Pilot postabortion projects and small-scale expansion programs generally operate with donated instruments, because they are conducted before the commercial importation or local manufacturing of instruments receives regulatory approval. This partly reflects the sociopolitical tensions surrounding abortion-related health care, which create extraordinary pressure to demonstrate medical benefits and acceptability of new clinical practices. Attention to ensuring sustained provision of manual vacuum aspiration is generally put off until after the program has achieved a degree of success and recognition.

Foundations' and donor agencies' generous donations of manual vacuum aspiration instruments have greatly eased the implementation of demonstration projects and research studies. However, the long-term viability of a program that relies on donated instruments is uncertain (at best), and a sustainable supply of instruments is crucial for a national program. In settings where access to abortion is legally re- stricted, it is extremely difficult to gain approval to purchase or manufacture the instruments, because of their association with abortion. Procurement decisions are frequently made by officials who are not clinicians or who are unfamiliar with the need for improved postabortion services. ${ }^{4}$ As a result, operational policies that govern the availability of manual vacuum aspiration instruments have changed more slowly than medical guidelines for providing postabortion care. Both are important for the expansion of pilot programs.

Three bodies of theoretical literature are relevant to understanding program expansion: those on the diffusion of innovation theory, ${ }^{5}$ public policy formation ${ }^{6}$ and knowledge management. ${ }^{7}$ Recent work has begun organizing these three streams into a single discussion. For example, Simmons and colleagues have identified seven elements of successful expansion programs, including allowing for adaptation and incremental change, working through the political process by adopting a highly pragmatic management style and engaging the support of one or more policymakers who are open to change. ${ }^{8}$ The Egyptian postabortion care experience is an example of how these elements contributed to the successful expansion of a small-scale activity into a national program, and the lessons learned are applicable to other settings.

\section{THE EGYPTIAN PROGRAM: INITIAL PHASES}

The few studies on Egyptian abortion practices reported during the $1970 s^{9}$ did not initiate a strong research tradition, and the growing importance of conservative socioreligious forces during the 1980s squelched interest in improving routine medical care for incomplete abortion. * This trend ran counter to changes in population programs in

*On a worldwide scale, Egypt's abortion policy is somewhat restrictive. The 1937 penal law (still in effect) explicitly prohibits acts that cause an abortion and stipulates stiff penalties for practitioners; it also contains provisions permitting any medical treatment necessary to save the life of a woman, which have been interpreted to justify abortion in life-threatening circumstances (source: Azer A, Law as an instrument for social change: an illustration from population policy, Cairo Papers in Social Science, 1979, Vol. 2, No. 4). In any case, the legal definition of abortion is strongly moderated by religious considerations. Egyptian Islamic theologians generally view abortion to save a woman's life as acceptable, although what constitutes a life-threatening condition is not clearly defined (sources: International Planned Parenthood Federation, Unsafe abortion and sexual health in the Arab world, paper presented at the conference Unsafe Abortion and Sexual Health in the Arab World, Damascus, Syria, Dec. 1992; and Omran A, Family Planning in the Legacy of Islam, New York: Routledge Press, 1972). As a result of conflicting legal provisions and differing theological opinions, abortion in Egypt remains a complex issue and a not uncommon practice (source: Huntington D, Abortion in Egypt: official constraints and popular practices, in: Makhlouf Obermeyer C, ed., Cross-Cultural Perspectives on Reproductive Health, New York: Oxford University Press, 2001).
By Dale

Huntington and Laila Nawar

Dale Huntington is senior health specialist, World Bank, Washington, DC; at the time this article was written, he was with the Population Council, Cairo and New Delhi. Laila Nawar is program associate, Population Council, Cairo. 
the years leading up to the 1994 International Conference on Population and Development, as operations researchers were being drawn into investigations of what constitutes (and how to deliver) an appropriate constellation of reproductive health care services.

As international dialogue opened in the early 1990s, Population Council researchers in Cairo began discussions with the minister of state for population and family planning about developing a program to study the routine care provided to women who have had an incomplete abortion and to improve service delivery by instituting changes that could be implemented fairly quickly. (This early contact with an influential government official known for having an interest in innovative policy was key to garnering support for the program.) Implicit in this discussion was an understanding that the rationale for studying postabortion services was to improve existing health care services, not to generate data for advocating greater access to induced abortion. This trust among researchers, program managers and policymakers has guided the program to the present.

A 1994-1995 operations research study ${ }^{10}$ was one of the first to reveal the substandard quality of care that is provided to postabortion patients and the feasibility of rapidly improving outcomes related to both providers (e.g., technical knowledge, surgical techniques and interpersonal communication skills) and patients (e.g., pain management and use of local anesthesia, knowledge about the timing of return to fertility and about warning signs of possible complications, and intention to practice contraception). Although the study generated considerable international interest, the research team purposely muted the communication of its results within Egypt. Staff felt that because postabortion services had virtually no constituency at that time (apart from the few senior government officials and hospital staff who took part in the pilot study), active dissemination of the study's results might have stimulated misperceptions and rumors, and violated the sense of trust regarding the objective of the research. Important findings, however, were communicated through a series of targeted briefings to appropriate government officials and important donor agencies in Egypt.

The 1994-1995 study led to medical improvements without causing public controversy; this, in turn, led to support for continuing work on postabortion services. Additionally, Population Council and Ipas staff collaborated to produce a strategy statement to guide the development of the postabortion care program, following the design of a program introducing contraceptive implants in Egypt. ${ }^{11}$ The first element of this strategy involved a small-scale expansion of services from the two sites of the pilot study into 10 district, general, university and teaching hospitals in upper and lower Egypt during 1996-1997.

Like the pilot program, the expansion project adopted

*The Packard Foundation has provided additional grants for instruments to the Population Council and other, national nongovernmental organizations working on improving postabortion care services in Egypt. These grants have ensured a steady supply of instruments. a management approach geared toward introducing service delivery improvements over a relatively short period of time. A mix of international and local experts provided intensive training for senior clinicians in each of the 10 hospitals, followed by extensive supervision of on-the-job clinical training of junior staff and nurses. The United Nations Population Fund provided a sufficient quantity of manual vacuum aspiration sets to last in the 10 sites for several years of services. * The program had a positive impact on providers' postabortion care knowledge and skills, and on patients' intentions to use family planning methods. ${ }^{12}$

As part of a generalized shifting of responsibilities within the Egyptian government that was under way during this period, oversight for the postabortion program moved to the Ministry of Health and Population after the pilot study. During this first phase of the program's expansion, it was still closely linked to the national family planning program in a number of ways: It was organized along the lines of a contraceptive introduction program, it emphasized providing contraceptives or referral for family planning services, and its funding came from the population sectors of the U.S. Agency for International Development and the Egyptian government. The goal of the expansion was to influence national policy by engaging high-level policy advisors. Population Council staff worked with the Ministry of Health to convene a technical advisory committee to review and approve training materials and norms of care for postabortion services. The expansion project also created a pool of experienced postabortion care clinicians, drawn from among leading Egyptian obstetrician-gynecologists.

Population Council staff collaborated with several agencies on a series of operations research studies concurrent with the expansion program, using the 10 hospitals as laboratories for testing alternative forms of postabortion service delivery ${ }^{13}$ and expanding the scope of inquiry to document both the magnitude of the health care problem caused by unsafe abortion in Egypt ${ }^{14}$ and women's perceptions of abortion. ${ }^{15}$ These studies contributed to the growing body of international evidence supporting the global postabortion care initiative during its formative years and created an environment for scientific study of postabortion services that engaged the Egyptian public health community.

The expansion program also worked to create formal linkages between the manufacturer that supplied manual vacuum aspiration instruments to the program (Ipas) and an Egyptian commercial agency that could apply for the rights to import and sell instruments in Egypt. Three applications were filed with the government's regulatory committee in 1996-1998, and each was rejected, despite the considerable body of international evidence and breadth of local experience gained through the expansion program regarding the need for manual vacuum aspiration equipment. The failure to secure approval for the commercial importation of the instruments can be attributed to the review board's unwillingness to approve any technology that is associated with induced abortion. Additionally, no highly influential policy advocate emerged on the issue of im- 
proving postabortion services, as both the personal and the professional risks of being perceived as lobbying for liberalization of restrictions on abortion were too great.

These dynamics could be addressed only by improving postabortion care and having manual vacuum aspiration become the usual and customary treatment for postabortion patients nationwide. However, broad-based familiarity with the postabortion program had not developed within the relatively short trial period. More time and greater programmatic experience were required to win further support for postabortion care.

\section{MOVING FROM RESEARCH TO PROGRAM}

A nascent group of postabortion care supporters, starting with the few clinicians who took part in the initial expansion, held a series of meetings to review research results and discuss knowledge gained from clinical experience. The meetings also stimulated public discussion of the postabortion care initiative, making the topic more acceptable. At the same time, the program's focus shifted from research toward clinical issues. Program managers within the Ministry of Health and hospital administrators also took part in the discussions, further broadening support for the program.

These highly participatory review meetings highlighted the important role of follow-up support to physicians after their initial training in postabortion care. Sustaining adherence to standard protocols that introduce an innovation is problematic in many clinical settings. Reports consistently indicated that physicians in Egyptian public hospitals were initially curious about the instruments for manual vacuum aspiration and the use of local anesthesia, but after a trial period, many would revert to their former practice of using dilation and curettage (D\&C) with general anesthesia. Initially, program managers thought that this was because of insufficient follow-up support or lax administrative controls over clinical practices. However, further experience with the program suggested a different reason. Because manual vacuum aspiration instruments were unavailable in the private sector, physicians used manual vacuum aspiration in public clinics (where intense monitoring helps ensure physicians' adherence to new case management protocols) and D\&C in their private practices. Given that most Egyptian physicians work in both public clinics and private settings, it was not surprising that they would favor developing proficiency in the technique used in their fee-for-service practices. Thus, the overall limited availability of manual vacuum aspiration instruments had a negative effect not only on the long-term viability of the expansion program but also on the immediate adoption of improved clinical practices related to postabortion care, even where instruments were available.

In addition to revealing constraints on sustaining improved clinical practices, these meetings indicated several shortcomings in the expansion program's original strategy. Because of the highly sensitive nature of abortion, the regulatory review and approval process for essential com- modities for the postabortion program was qualitatively different from that for family planning program commodities. Furthermore, the national family planning program could not provide the resources required for the obstetric training that physicians needed to provide postabortion care. In any case, national family planning program managers were not well placed to oversee such improvements. The service delivery model used by postpartum family planning programs was explored and found to be inadequate for postabortion care, because postpartum family planning programs typically are not involved in improving the obstetric care that precedes the provision of a contraceptive method. Improving emergency medical treatment for incomplete abortion is a critically important element of postabortion care, and working only from within the population sector, it was not possible to devote sufficient resources, or engage the appropriate constituencies, to achieve this objective.

\section{INTEGRATION INTO THE SAFE MOTHERHOOD PROGRAM}

Improvements in obstetric services fall within the domain of maternal health programs. The Ministry of Health and Population has long been aware of the role of unsafe abortion in causing maternal deaths, ${ }^{*}$ and its Directorate of Maternal and Child Health administers the nationwide Safe Motherhood Program (the Healthy Mother/Healthy Child Project ${ }^{\dagger}$ ), a set of interventions designed to reduce maternal mortality. Results from the project's needs assessments ${ }^{16}$ showed an absence of ministry-approved clinical protocols for managing abortion complications. Staff from the Directorate of Maternal and Child Health participated in some of the meetings that reviewed the postabortion expansion program and began discussions with Population Council staff about incorporating elements of the postabortion care service delivery package within the government's Essential Obstetric Care Program.

The Ministry of Health and Population's essential obstetric care protocols ${ }^{17}$ are based on the results of postabortion care research in Egypt and on worldwide experience. Within the guidelines for managing bleeding in pregnancy (hemorrhage is the leading cause of maternal mortality in Egypt ${ }^{18}$ ), they specify manual vacuum aspiration as the procedure of choice in the treatment of incomplete abortion at less than 20 weeks' gestation. The protocols also emphasize other elements of postabortion care, including the use of local anesthetics in combination with analgesics, anxiolytics or sedatives; the importance of interpersonal communication and counseling about warning signs and

*According to the National Maternal Mortality Surveys, $5 \%$ of maternal deaths in 1992-1993 and 4\% in 2000 were due to abortion-related complications; $11 \%$ of pregnancies ended in spontaneous abortion in 2000 (source: Ministry of Health and Population, Egypt: National Maternal Mortality Study 2000, Cairo: Directorate of Maternal and Child Health Care, 2001). The national postabortion caseload study revealed that one out of five obstetric-gynecologic admissions are related to incomplete abortion (source: reference 14).

†The project is implemented by the Ministry of Health and Population, with technical assistance from John Snow, Inc., and support from the United States Agency for International Development. 
recuperation; aseptic techniques and procedures; and referral for related reproductive health services.

Significantly, the protocols do not use the term "postabortion" and do not emphasize the integration of family planning counseling. In part, the omission of family planning reflects that the guidelines are limited to the clinical management of complications and do not address preventive health care practices (including using contraceptives to avoid an unwanted pregnancy). It also reflects the history of the family planning program as a vertically funded and managed one. Linkages between the national Safe Motherhood and family planning programs are a stated goal of the Ministry of Health and Population, yet little has been done to combine these services.

The Egyptian Safe Motherhood Program's use of the postabortion care service package represents the jump from research to program that could take the postabortion initiative to national scale. Although this is a tremendous advance, programmatic experience with the essential obstetric care protocols indicates that unresolved issues in sustaining improvements in postabortion care continue to bedevil the national program. The Essential Obstetric Care Program relies upon donated manual vacuum aspiration instruments, and the commercial sale of instruments in Egypt has not been approved. The tendency for physicians to revert to using D\&C is still evident, although it is driven by more than simply a lack of instruments available for use in private practice settings. For example, internal reviews suggest that in its introductory activities, the Essential Obstetric Care Program did not focus sufficiently on increasing the skills and redefining the role of nursing staff, which has hampered staff's ability to ensure that aseptic manual vacuum aspiration sets and necessary medication are ready for use in the operating theater. ${ }^{19}$ In addition, the small caseload in district-level facilities does not provide sufficient opportunity to practice with the new protocols; as a result, an extended period of follow-up support is required, and this challenges the abilities of Ministry of Health and Population's routine supervisory systems.

\section{CONCLUSIONS}

As it moved from inception to a national program, the Egyptian postabortion care initiative used a highly flexible, innovative management style that was consistent with contemporary theories of how to diffuse innovation and scale up pilot activities. During the pilot study and initial expansion, the program maintained a strictly defined sense of identity as a postabortion research activity, achieving rapid success and demonstrating promise that drew the attention of innovative managers and policymakers. However, in this phase, the program's replication was limited to carefully controlled circumstances, and the program failed to create circumstances for its scaling-up: It did not create broad enough partnerships or integrate the new services into an existing program. Furthermore, the strategy modeled on family planning programs' strategy for introducing new contraceptives was not functional for postabortion care, be- cause of sensitivities regarding abortion.

As an active communication program brought together larger and larger groups of clinicians, and Ministry of Health and Population managers spurred dialogue about the clinical experience and knowledge gained, strategies for how to proceed were identified. Ownership of the postabortion program passed to the national Safe Motherhood Program, which incorporated most elements of the service delivery package and did away with the term "postabortion care." This process of unbundling and relearning was essential to the integration of the postabortion care model into a larger program. Postabortion programs in other settings can learn from this experience and seek out partnerships with national programs that are working to improve maternal health services.

The experience with the Egyptian postabortion care program also provides insights into policy-making associated with highly sensitive services. Postabortion initiatives will never be able to entirely avoid the politically charged atmosphere unique to abortion, particularly in settings where access to induced abortion services is legally restricted. Further, it is unlikely that a single policy advocate (or a committed few) will take a public stance and push for postabortion care-related reforms. Although the research program benefited from the support of farsighted decision-makers within the government and the donor community, expansion into a national program occurred only in the context of a larger effort to improve all types of obstetric services, and was not the result of a policy change specific to postabortion care. In such contexts, decisions related to abortion are made very slowly, and service delivery innovations must become usual and customary practices before a policy will change. The lag in critically important operational policy decisions (e.g., approval of the commercial importation of manual vacuum aspiration instruments) requires the long-term commitment of the program's international supporters. This approach to influencing policymaking is qualitatively different from the approach taken for other health care topics, and reflects a profound understanding of the politics of abortion, rather than an avoidance of the political process.

Clearly, a program will make many trade-offs and compromises as it moves from research to small-scale expansion and then to a national program. In this respect, the scaling-up of postabortion care services is no different from the scaling-up of other programs. Family planning programs cannot provide postabortion initiatives with the support that is required for going to scale with improvements in emergency medical services, and Safe Motherhood programs have a limited ability to provide contraceptive-related services. Postabortion care straddles these programs and ultimately will be realized only as holistic programming is achieved for women's reproductive health services.

\section{REFERENCES}

1. Langer A et al., Improving postabortion care with limited resources in a public hospital in Oaxaca, Mexico, in: Huntington D and Piet-Pelon NJ, eds., Postabortion Care: Lessons from Operations Research, New York: 
Population Council, 1999; Solo J et al., Creating linkages between incomplete abortion treatment and family planning services in Kenya, Studies in Family Planning, 1999, 30(1):17-27; and Huntington D et al., Improving the medical care and counseling of postabortion patients in Egypt, Studies in Family Planning, 1995, 26(5):350-362.

2. Greenslade FC et al., Manual Vacuum Aspiration: A Summary of Clinical and Programmatic Experience Worldwide, Carrboro, NC, USA: Ipas, 1993.

3. Diaz J et al., Improving the quality of services and contraceptive acceptance in the postabortion period in three public sector hospitals in Bolivia, in: Huntington D and Piet-Pelon NJ, 1999, op. cit. (see reference 1).

4. Cross $\mathrm{H}$ et al., Reforming Operational Policies: A Pathway to Improving Reproductive Health Programs, Policy Occasional Paper, Washington, DC Futures Group International, 2001, No. 7.

5. Rogers EM, Diffusion of Innovations, New York: Free Press, 1995.

6. Christoffel KK, Public health advocacy: process and product, American Journal of Public Health, 2000, 90(5):722-726; Mintrom M, Policy entrepreneurs and the diffusion of innovation, American Journal of Political Science, 1997, 41(3):738-770; and Porter RW and Hicks I, Knowledge Utilization and the Process of Policy Formation: Toward a Framework for Africa, Washington, DC: Academy for Educational Development, 2001.

7. Sveiby KE, The New Organizational Wealth: Managing and Measuring Knowledge-Based Assets, San Francisco, CA, USA: Berrett-Koehler Publishers, 1997; and Nonaka I and Takeuchi H, The Knowledge-Creating Company, New York: Oxford University Press, 1995.

8. Simmons R et al., Facilitating large scale transitions to quality of care: an idea whose time has come, Studies in Family Planning, 2002, 23(1): $61-75$.

9. Foda MS et al., Induced Abortion: A Hazard to Public Health? Beirut, Lebanon: International Planned Parenthood Federation (IPPF), 1971; Suliman N, Abortion in Egypt, Population Studies, Beirut, Lebanon: IPPF, 1979, No. 49; and Kamal I et al., Epidemiological Study of Induced Abortion by Pregnancy Screening in a Rural Community in Egypt, 1972-1973, Cairo: Supreme Council for Population and Family Planning, 1975.
10. Huntington D et al., Improving the medical care and counseling of postabortion patients in Egypt, Studies in Family Planning, 1995, 26(5):350362.

11. Huntington D and Greenslade F, Strategy statement: introduction program for postabortion medical care services in Egypt, Cairo: Population Council, 1994.

12. Nawar L and Huntington D, Expanding improved postabortion care is underway, paper presented at the general conference of the International Union for the Scientific Study of Population, Beijing, Oct. 11-17, 1997.

13. Abdel-Tawab $\mathrm{N}$ et al., Effects of husband involvement on postabortion patients' recovery and use of contraception in Egypt, in: Huntington D and Piet-Pelon NJ, op. cit., 1999 (see reference 1).

14. Huntington D et al., The postabortion caseload in Egyptian hospitals: a descriptive study, International Family Planning Perspectives, 1998, 24(1): 25-31.

15. Huntington D, Nawar L and Abdel-Hady D, Women's perceptions of abortion in Egypt, Reproductive Health Matters, 1997, 5(9):101-107.

16. Megeid AA, John Snow, Inc., Cairo, personal communication, July 2002; and Shoubary AA, El Gala Hospital, Cairo, personal communication, July 2002.

17. Ministry of Health and Population (MOHP), Essential Obstetric Care: Protocol for Physicians, Cairo: Directorate of Maternal and Child Health Care, MOHP, 2001; and MOHP, Manual Vacuum Aspiration, Essential Obstetric Care: A Competency-Based Training Manual for Physicians, Cairo: Directorate of Maternal and Child Health Care, MOHP, 2001.

18. MOHP, Egypt: National Maternal Mortality Study 2000, Cairo: Directorate of Maternal and Child Health Care, MOHP, 2001

19. Megeid AA, 2002, op. cit. (see reference 16); and Shoubary AA, 2002, op. cit. (see reference 16).

Author contact: dhuntington@worldbank.org 